\title{
Acute Abdominal Pain
}

\author{
Thamilselvam $\mathbf{P}^{* 1}$ and Vinoth Kumar $\mathbf{R}^{2}$ \\ ${ }^{1}$ Department of Surgery, National defence University of Malaysia, Malaysia \\ ${ }^{2}$ Department of Urology, Aarupadai veedu Medical college, India
}

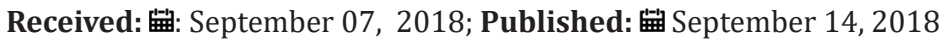

*Corresponding author: Thamilselvam P, Department of Surgery, Head of Department, National defence University of Malaysia, Malaysia

\section{Abstract}

The treatment for pain before arriving the diagnosis in patients with acute abdominal pain still remains controversial. Many recent studies have showed that the treatment of pain does not negatively influence either the diagnosis or subsequent treatment of these patients; however, current practice patterns continue to favour withholding pain medication prior to diagnosis and surgical treatment decision [1]. Pain is a complex phenomenon with various causes and issues associated with its incidences. This complexity is especially true for those who have chronic pain. In light of the multifactorial nature of this problem, the treatment plan has to be individualized for each patient [2]. Any doctor doing practice in emergency medicine should be skilled in the assessment of abdominal pain and related diseases. Although a common presentation, abdominal pain must be approached in a serious manner, as it is often a symptom of serious disease and misdiagnosis may occur.

Abdominal pain is the presenting issue in a high percentage of medico legal actions against both general and paediatric emergency medicine physicians [3,4]. The modern physician should be humbled by the fact that, despite diagnostic and therapeutic advances (computed tomography, ultrasonography, interventional radiology and laparoscopy), the misdiagnosis rate of the most common surgical emergency, acute appendicitis, has changed little over time [5]. Our purpose of this article is to help and provide clinicians with a framework for evaluating the complaint of acute abdominal pain and recognizing patients who require expedited evaluation for the treatment and further management.

\section{Mini Review}

\section{Abdominal Pain Conditions}

There are some of the most common causes of abdominal pain are inflammation of appendix, gallbladder, Pancreatitis, diverticulitis, Gastroesophageal reflux disease, small and large bowel 's diseases and it's obstruction and renal colic.

\section{Appendicitis}

This is the commonest condition of hospital admissions and surgeries around the world. There are 250,000 appendectomies performed in the United States every year [6,7]. The diagnosis of appendicitis needs to be made quickly because the risk for rupture or perforation increases significantly after 36 hours from the onset of symptoms [8]. The typical presentation of appendicitis includes a patient complaining of periumbilical, colicky pain that worsens during the first 24 hours. The pain then localizes to the right lower quadrant of the abdomen and sharpens in intensity. This migration of pain is the most consistent finding for appendicitis $[9,10]$. Most of the surgeons diagnose the acute appendicitis by clinical and do some investigations to rule out other acute abdominal conditions. Although appendicitis is the most common reason to undergo surgery due to right iliac fossa pain, there are many other diseases which can mimic or confuse the picture, including diverticulitis, mesenteric inflammation, Crohn's disease, infectious enterocolitis, endometriosis, ectopic pregnancy, and intussusception, to name a few [11]. It is advisable to avoid analgesic and sedation till we arrive the proper diagnosis of acute appendicitis , otherwise it would lead to the wrong diagnosis since the pain in right iliac fossa would have been masked.

\section{Acute Cholecystitis}

Patients with right hyphochondrial pain with fever is the commonest presentation for acute cholecystitis. Acute cholecystitis-inflammation of the gall bladder-is most often caused by gall stones. Gall stones are one of the most common disorders of the gastrointestinal tract, affecting about $10 \%$ of people in Western society [12,13]. More than $80 \%$ of people with gall stones are asymptomatic. Acute cholecystitis develops in 1-3\% of patients with symptomatic gall stones [14]. Ultrasound abdomen is the best key investigation to arrive the diagnosis. Adrian A, Indar et all stated as below

a. Patients suspected of having acute cholecystitis should be referred to hospital immediately.

b. First line treatment includes fasting, intravenous fluids and analgesia.

c. Surgery (cholecystectomy) within 24-48hours of admission (early) as preferable to delayed or "interval" surgery. 
d. Percutaneous cholecystostomy is a safe alternative to cholecystectomy for very ill patients or those unfit to undergo surgery.

e. In $20 \%$ of cases, emergency surgery is needed to treat gangreneous cholecystitis or gall bladder perforation [15].

There are some evidences showing that chronic inflammation of gallbladder would cause carcinoma gallbladder. Chronic inflammation of the gallbladder for 15 or more years in a genetically predisposed individual likely promotes malignant transformation, aided in some instances by exposure to carcinogens. Carcinoma of the gallbladder is a multistep process involving cumulative genetic and epigenetic alterations that include activation of oncogenes and inactivation of tumor suppressor genes [16].

\section{Acute Diverticulitis}

It is an inflammation of diverticular disease of colon with clinical presentation of pain abdomen. The site of pain varies according to part of colon involved and the pain would be generalised if the diverticulitis gets perforated. The common site of diverticulum is sigmoid colon and the past history of passing pellet like stool would support the diagnosis. On clinical examination, there would be tenderness at diverticulitis area. There would be pnemo peritoneum in plain X-ray abdomen, if there was perforated diverticulitis. As reported above, today contrast-enhanced CT has a pivotal role in the clinical practice regarding diverticular disease, and because of their superior sensitivity and specificity up to $100 \%$, replaced the most important imaging modality [17]. Especially when an associated abscess is suspected, a CT scan can be very helpful to demonstrate its presence, and also for evaluate the possibility of a percutaneous drainage [18].

\section{Acute Pancreatitis}

Acute pancreatitis is a chemical inflammation of the pancreas and sometimes associated with a systemic inflammatory response which can impair the function of other vital organs. It may settle sometimes or it may lead to necrosis of the pancreas and surrounding fatty tissue with severe sepsis. Acute pancreatitis is a common disease with a high mortality [19], and frequently caused by gallstone disease [19] or excess alcohol ingestion [20]. The diagnosis of acute pancreatitis is supported by an elevation of the serum amylase and lipase levels. The amylase level becomes elevated within hours of the development of pain and may remain elevated for 3 to 5 days. There are some other conditions as well where there is raise in serum amylase and these are

a. Post Endoscopic Retrograde Cholangio Pancreatogram (ERCP).

b. Post-surgical (Stomach and splenic surgeries).

c. Blunt injury abdomen.

Serum lipase has higher specificity for pancreatic disease, but its level may be elevated in other conditions as well. Laboratory abnormalities encountered in acute pancreatitis include hyperglycemia, hypocalcemia, leukocytosis, and mild elevations of liver function test results. Ultrasound and magnetic resonance cholangiopancreatography are potentially valuable tests in the evaluation of acute pancreatitis and are helpful in detecting stones in the common bile duct and directly assessing the pancreatic parenchyma [21].

\section{Gastroesophageal Reflux Disease}

Gastroesophageal reflux disease is commonly managed in both primary and secondary care settings, as this condition occurs in patients of all ages and has a wide variety of clinical presentations. However, evidence suggests that Gastroesophageal reflux disease is commonly overdiagnosed and overtreated. Adherence to guidelines may help reduce the harms of overdiagnosis [22]. Physician's role is very important to rule out ischaemic heart disease which would mimic the signs and symptoms of Gastroesophageal reflux disease.

\section{Treatment}

Lifestyle modification is often recommended for the treatment of GERD symptoms. For nocturnal symptoms, elevating the head of the bed and avoiding meals 2-3 hours prior to bedtime may be helpful. For patients with a body mass index above $25 \mathrm{~kg} / \mathrm{m} 2$ or patients with a normal body mass index but recent weight gain, weight loss may result in significant improvement of symptoms [23]. Other commonly touted lifestyle recommendations such as avoidance of caffeine, carbonated beverages, alcohol, spicy foods, and fatty foods have no evidence to support them [24]. Proton pump inhibitors have the main role to reduce the acidity in stomach and PPI would heal the mucosal inflammation and erosions of Oesophago gastric junction and lower end of oesophagus.

\section{Ulcerative Colitis}

Ulcerative colitis is a chronic, inflammatory bowel disease that arises from an interaction between genetic and environmental factors [25] .The primary presenting symptom of Ulcerative colitis is visible blood in the stools, which is reported by more than $90 \%$ of Ulcerative colitis patients 25 However, since the fecal occult blood test and colonoscopy have recently been used for colorectal cancer screening as part of general health screening programs, a small number of Ulcerative colitis patients that lack Ulcerative colitis -related symptoms are diagnosed during screening examinations [26]. The signs and symptom are diarrhea, crampy abdominal pain, bloody diarrhea with mucus, weight loss, loss of appetite, tiredness, anemia and fever. Treatment includes amino salicylates, steroids and azathioprine (immune suppressant ). If the medical line of management of Ulcerative colitis fails, the surgical treatment is indicated to prevent further complications namely colonic cancer , toxic mega colon and fistulae.

\section{Ischemic Colitis}

The patients are with severe abdominal pain with bleeding per rectum. The patients with poor prognostic disease can be saved by arriving early diagnosis by doing CT Angiogram. CT angiogram was performed in more than a quarter of our patients following the diagnosis of ischemic colitis. CT angiogram detected more stenosis than the contrast-enhanced CT scan as the primary goal of performing the latter is not for detecting this finding. However, CT angiogram did not change the management or the prognosis of ischemic colitis. We recommend against the routine use of CT angiogram after the diagnosis of ischemic colitis. CT angiogram 
might be considered in more severe cases of ischemic colitis especially with right colon involvement or in instances of the need for excluding acute mesenteric ischemia based on clinical scenarios [27].

\section{Acute Intestinal Obstruction}

This is also very common condition which can be easily diagnosed. The patients are with pain abdomen, vomiting, abdominal distension and constipation and the clinician in emergency department has to start intravenous fluids and insert ryles tube immediately. The patient has to be ordered for X-ray abdomen which would give information not only for diagnosis but also for the plan of the management. Bowel obstruction continues to be one of the most common abdominal problems faced by general surgeons. Irrespective of the cause, it remains a major cause of morbidity and mortality. Success in the treatment of intestinal obstruction depends largely upon early diagnosis, skilful management and treating the pathological effects of the obstruction just as much as the cause itself. Early recognition and aggressive treatment are crucial in preventing irreversible ischemia and transmural necrosis and thereby in decreasing mortality and longterm morbidity. The evaluation of patients with suspected bowel obstruction endeavours not only to confirm the diagnosis but also to determine the need for and timing of surgery. Certain severity indicators and scoring systems can help to optimize this timing of surgery and prevent mortality [28,29].

\section{Renal Colic}

Renal colic and ureteric colic have acute radiating pain over loin and it may be associated with painful or painless haematuria. Sever loin pain with sweating may be the main symptom. Urine examination and ultra sound KUB would help to arrive the diagnosis and further management. The commonest cause for the renal colic is stones. Nephrolithiasis remains a major economic and health burden worldwide. Nephrolithiasis is considered a systemic disorder associated with chronic kidney disease, bone loss and fractures, increased risk of coronary artery disease, hypertension, type 2 diabetes mellitus, and the metabolic syndrome. Further understanding of the pathophysiological link between nephrolithiasis and these systemic disorders is necessary for the development of new therapeutic options.

\section{Conclusion}

There are lot of causes for acute abdomen and we discussed here some of the conditions only. We have to get proper history and do physical examination. We have to order relevant investigations to arrive proper diagnosis for further treatment. In acute abdomen patients, we need not follow regular procedures as investigations first and treatment later. We need to start the acute management (IV fluids, blood transfusion and antibiotics) simultaneously when we order for the investigations.

\section{References}

1. Falch C, Vicente D, Häberle H, Kirschniak A, Müller S, et al. (2014) Treatment of acute abdominal pain in the emergency room: a systematic review of the literature. Eur J Pain 18(7): 902-913.
2. Scott F Nadler (2004) Non-pharmacologic management of pain. The Journal of the American Osteopathic Association 104(8): 6S-12S.

3. Selbst SM, Friedman MJ, Singh SB (2005) Epidemiology and etiology of malpractice lawsuits involving children in US emergency departments and urgent care centers. Pediatr Emerg Care 21(3): 165-169.

4. Kachalia A, Gandhi TK, Puopolo AL (2007) Missed and delayed diagnoses in the emergency department: a study of closed malpractice claims from 4 liability insurers. Acad Emerg Med 49(2): 196-205.

5. Flum DR, Morris A, Koepsell T (2001) Has misdiagnosis of appendicitis decreased over time? JAMA 286(14): 1748-1753.

6. Addiss DG, Shaffer N, Fowler BS, Tauxe RV (1990) The epidemiology of appendicitis and appendectomy in the United States. Am J Epidemiol 132(5): 910-925.

7. Shelton T, McKinlay R, Schwartz RW (2003) Acute appendicitis: current diagnosis and treatment. Curr Surg 60(5): 502-505.

8. Bickell NA, Aufses AH, Rojas M, Bodian C (2006) How time affects the risk of rupture in appendicitis. J Am Coll Surg 202(3): 401-406.

9. Andersson RE (2004) Meta-analysis of the clinical and laboratory diagnosis of appendicitis. Br J Surg 91(1): 28-37.

10. Wagner JM, McKinney WP, Carpenter JL (1996) Does this patient have appendicitis? JAMA 276(19): 1589-1594.

11. Purysko AS, Remer EM, Filho HM, Bittencourt LK, Lima RV, et al. (2011) Beyond appendicitis: common and uncommon gastrointestinal causes of right lower quadrant abdominal pain at multidetector CT. Radiographics 31(4): 927-947.

12. Jensen KH, Jorgensen $\mathrm{T}$ (1991) Incidence of gallstones in a Danish population. Gastroenterology 100(3): 790-794.

13. Bates T, Harrison M, Lowe D, Lawson C, Padley N (1992) Longitudinal study of gall stone prevalence at necropsy. Gut 33(1): 103-107.

14. Friedman GD (1993) Natural history of asymptomatic and symptomatic gallstones. Am J Surg 165(4): 399-404.

15. Adrian AI, Ian J B (2002) Acute cholecystitis. BMJ 325(7365): 639-643.

16. Eldon A Shaffer (2008) Gallbladder Cancer. Gastroenterol Hepatol (NY) 4(10): 737-741.

17. Le Pennec V, Hourna E, Schmutz G, Pelage JP (2012) Imaging in infections of the left iliac fossa. Diagn Interv Imaging 93(6): 466-472.

18. Brandt D, Gervaz P, Durmishi Y, Platon A, Morel P, et al. (2006) Percutaneous CT scan- guided drainage vs. antibiotherapy alone for Hinchey II diverticulitis: a case-controlstudy. Dis Colon Rectum 49(10): 1533-1338.

19. Jovicic I, Petronijević L, Denić L, Golubović G, Kontić M (2009) Epidemiology of acute pancreatitis in Belgrade. Pancreatology 9: 510.

20. Munsell MA, Buscaglia JM (2010) Acute Pancreatitis. J Hosp Med 5: 241250.

21. Bo Guang F, Åke AS (2010) Acute Pancreatitis. N Am J Med Sci 2(5): 211214.

22. J Lane W (2016) Gastroesophageal Reflux Disease. Treating Wisely North Carolina Medical Journal 77(3): 202-205.

23. (2014) Drugs for peptic ulcer disease and GERD. Treat Guidel Med Lett 12(140): 25-30.

24. Katz PO, Gerson LB, Vela MF (2013) Guidelines for the diagnosis and management of gastroesophageal reflux disease. Am J Gastroenterol 108(3): 308-328.

25. Dignass A, Eliakim R, Magro F, Maaser C, Chowersn Y, Geboes K, et al. (2012) Second European evidence-based consensus on the diagnosis and management of ulcerative colitis part 1: definitions and diagnosis.J Crohn's Colitis 6(10): 965-990. 
26. Both H, Torp Pedersen K, Kreiner S, Hendriksen C, Binder V (1983) Clinical appearance at diagnosis of ulcerative colitis and Crohn's disease in a regional patient group. Scand J Gastroenterol 18(7): 987-991.

27. Sherid M, Samo S, Sulaiman S, Husein H, Sethuraman SN, et al. (2014) Is CT Angiogram of the Abdominal Vessels Needed following the Diagnosis of Ischemic Colitis? A Multicenter Community Study. ISRN Gastroenterology Volume 2014: 756926.

\section{ISSN: 2574-1241}

DOI: 10.26717/BJSTR.2018.09.001737

Thamilselvam P. Biomed J Sci \& Tech Res

(C) This work is licensed under Creative

Submission Link: https://biomedres.us/submit-manuscript.php
28. Saurabh J Tiwari (2017) A clinical study of acute intestinal obstruction in adults-based on etiology, severity indicators and surgical outcome. International Journal of Research in Medical Sciences 5(8): 3688-3696.

29. Khashavar S, Naim M (2012) Kidney Stones 2012: Pathogenesis, Diagnosis, and Management. J Clin Endocrinol Metab 97(6): 1847-1860.

Assets of Publishing with us
$\begin{aligned} & \text { BIOMEDICAL } \\ & \text { RESERCHES }\end{aligned}$
- Global archiving of articles

